Reprod. Nutr. Dévelop., 1988, 28 Suppl. n 1, 97-98

\title{
Effet de l'éthanol sur les fermentations dans un fermenteur semi-continu de type Rusitec
}

\author{
Andrée DURIX, T. MEZIANE, J. P. JOUANY ( $\left.{ }^{*}\right)$, C. JEAN-BLAIN
}

Laboratoire de Nutrition et Alimentation. Ecole Nationale Vétérinaire de Lyon 1, avenue Bourgelat, B.P. 83, 69280 Marcy-l'Etoile, France.

$\left(^{*}\right)$ I.N.R.A., Laboratoire de la Digestion,

Theix, 63122 Ceyrat, France.

Summary. The addition of ethanol increased acetate and methane production in a Rusitec fermentor, at the expense of propionate and $\mathrm{CO}_{2}$. Ethanol was fermented at least in part, but did not modify the disappearance of lucerne and sugar beet pulp. The microbial protein yield was improved to a large extent when $4 \mathrm{~g} / \mathrm{d}$ ethanol were given.

Certains aliments des ruminants (ensilages, drèches, etc...) peuvent contenir des quantités non négligeables d'éthanol, lequel peut être par ailleurs un produit mineur de la fermentation des glucides dans le rumen. L'effet de l'éthanol sur les fermentations intra-ruminales n'ayant fait l'objet que d'études de courte durée en systèmes in vitro, il a été étudié ici dans un fermenteur semi-continu de type Rusitec.

Matériel et méthodes. Un fermenteur ensemencé avec un inoculum provenant de 4 moutons fistulés nourris avec une ration mixte (paille + foin + luzerne déshydratée + orge) a été utilisé au cours de 3 périodes de 7 jours, faisant suite à une semaine d'adaptation : période 1 : aliment seul ; période 2 : aliment + forte quantité d'éthanol ( $8 \mathrm{~g}=174 \mathrm{mmoles}$ par jour) ajoutée en 2 fois; période 3 : aliment + faible quantité d'éthanol $(4 \mathrm{~g}=87$ mmoles par jour) ajoutée en 2 fois. Les aliments : $12 \mathrm{~g}$ de foin de luzerne, $5 \mathrm{~g}$ de pulpes de betteraves déshydratées, introduits séparément dans des sacs de nylon (mailles de $50 \mu$ ) sont retirés après un séjour de $48 \mathrm{~h} ; 700 \mathrm{ml}$ d'une solution tampon additionnés de $0,7 \mathrm{~g}$ d'urée et de $0,1 \mathrm{~g}$ de $\mathrm{Na}_{2} \mathrm{SO}_{4}$ sont infusés quotidiennement.

Nous avons mesuré les principaux paramètres fermentaires: acides gras volatils (AGV), matière organique fermentée (MOF), dégradation des parois cellulaires (dNDF) et de la lignocellulose (dADF), matière organique apparemment dégradée (MOAD). La protéosynthèse microbienne est estimée par différence entre l'azote total et l'azote ammoniacal $\left(\mathrm{N}=\mathrm{N}_{\mathrm{T}}-\mathrm{N}_{\mathrm{NH} 3}\right)$.

Résultats et discussion. L'éthanol augmente la production totale des acides gras volatils et modifie leurs proportions relatives. Le pourcentage molaire d'acétate augmente alors que celui de propionate diminue dans tous les cas. Par contre une forte élévation de la proportion d'isovalérate, de valérate et de caproate n'est notée qu'avec un faible apport d'éthanol ( $4 \mathrm{~g} / \mathrm{j}$ ) (tabl. 1). La MOF est augmentée avec l'apport élevé d'alcool mais ceci résulte de la transformation de 
l'éthanol en AGV et non d'une augmentation de la dégradation de la luzerne et de la pulpe de betteraves. En effet, les paramètres rendant compte de la dégradation de ces deux aliments (MOAD, d NDF et ADF) ne sont pas modifiés (tabl. 1). La production de méthane augmente avec l'apport élevé d'alcool, ce qui entraîne une diminution du rapport $\mathrm{CO}_{2} / \mathrm{CH}_{4}$ (tabl. 1). La quantité d'azote de la phase liquide $(\mathrm{N})$ et l'efficacité de la protéosynthèse $(\mathrm{Ng} / \mathrm{MOF} \mathrm{kg}$ ) doublent avec $4 \mathrm{~g}$ d'éthanol, alors qu'elles sont diminuées avec $8 \mathrm{~g}$ (tabl. 1).

TABL. 1. - Influence des différentes doses d'éthano/ sur la fermentation, les quantités de matière organique fermentée (MOF) ou apparemment disparue (MOAD), la disparition des parois cellulaires (d NDF) ou de la lignocellulose (d ADF) et la quantité de la phase liquide.

\begin{tabular}{|c|c|c|c|c|c|c|c|}
\hline $\begin{array}{l}\text { Période } \\
\text { Ethanol } \mathrm{g} / \mathrm{j}\end{array}$ & $\begin{array}{l}1 \\
0\end{array}$ & $\begin{array}{l}3 \\
4\end{array}$ & $\begin{array}{l}2 \\
8\end{array}$ & & $\begin{array}{l}1 \\
0\end{array}$ & $\begin{array}{l}3 \\
4\end{array}$ & $\begin{array}{l}2 \\
8\end{array}$ \\
\hline $\mathrm{pH}$ & 6,99 & 6,90 & 6,89 & $\mathrm{~N} \mathrm{mg} / \mathrm{j}$ & $133,4^{a}$ & $201,3^{b}$ & $101,8^{a}$ \\
\hline $\mathrm{AGV}$ totaux $\mathrm{mM} / \mathrm{j}$ & $70,7^{a b}$ & $75,7^{b}$ & $99,4^{a}$ & $\mathrm{Ng} / \mathrm{MOF} \mathrm{kg}$ & $20,3^{a}$ & $34,7^{b}$ & $11,4^{\mathrm{C}}$ \\
\hline Acétate & $60,9^{a}$ & $64,4^{c}$ & $69,7^{b}$ & MOAD & 6,2 & 6,4 & 6,5 \\
\hline Propionate & $23,7^{a}$ & $11,7^{c}$ & $16,2^{b}$ & d NDF (L) & 37,5 & 38,8 & 42,2 \\
\hline Isobutyrate \% & $0.7^{a}$ & $0,6^{\mathrm{c}}$ & $0,5^{b}$ & $(P)$ & 91,8 & 85,1 & 89,1 \\
\hline Butyrate molaire & 9,3 & 9,5 & 9,1 & d ADF (L) & 26,4 & 27,6 & 31,1 \\
\hline Isovalérate & $2,6^{a}$ & $3,7^{\mathrm{C}}$ & $0,8^{\mathrm{b}}$ & $(P)$ & 86,9 & 77,7 & 84,3 \\
\hline Valérate & $2,5^{\mathrm{a}}$ & $4,7^{\mathrm{b}}$ & $2,0^{a}$ & Volume gaz (ml) & 2852 & 2515 & 3077 \\
\hline Caproate & $0,3^{a}$ & $5,3^{c}$ & $0,9^{\mathrm{b}}$ & $\mathrm{CH}_{4} \%$ & $25,3^{a}$ & $24,6^{\mathrm{a}}$ & $30,4^{b}$ \\
\hline $\mathrm{MOF} g / \mathrm{j}$ & $6,4^{a}$ & $6,4^{a}$ & $9,0^{\mathrm{b}}$ & $\mathrm{CO}_{2} / \mathrm{CH}_{4}$ & $2,7^{\mathrm{a}}$ & $2,9^{a}$ & $2,2^{\mathrm{b}}$ \\
\hline
\end{tabular}

$\mathrm{L}$ : luzerne, $\mathrm{P}=$ pulpes de betteraves. Les valeurs affectées de lettres différentes sont significativement différentes entre elles au seuil $P \leqslant 0,05$.

L'éthanol est donc partiellement transformé en acides gras volatils dans le rumen. II ne modifie pas la dégradation des aliments solides qui lui sont associés. Par contre à faible dose il augmente le rendement de la protéosynthèse microbienne, très probablement en servant de source d'énergie aux microorganismes et en augmentant l'apport d'équivalents réduits.

A forte dose, il a un effet inhibiteur sur ce métabolisme. Ceci est en accord avec les données de Ørskov, Hemken et Moore (1967) et de Pradhan et Hemken (1970).

Ørskov E. R., Hemken R. W., Moore L., 1967. J. Dairy Sci., 50, 692-695.

Pradhan K., Hemken R. W., 1970. J. Dairy Sci., 53, 131-146. 